\title{
Discurso pedagógico en maestros desde lo enunciado por estudiantes de colegios católicos*
}

\author{
DIANA MARCELA PEDRAZA DÍAZ \\ dianapedrazadiaz@gmail.com
}

Recepción: 17 de febrero de 2015

Aprobación: 15 de abril de 2015

Forma de citar este artículo: Pedraza Díaz, D. (2015). Discurso pedagógico en maestros desde lo enunciado por estudiantes de colegios católicos. Cuadernos de Lingüística Hispánica, 26, 199-214, Tunja: Uptc.

\footnotetext{
Artículo de investigación científica, de corte pedagógico.
}

** Normalista Superior de la Escuela Normal de Bucaramanga, licenciada en Educación Básica con Énfasis en Lengua Castellana de la Universidad Industrial de Santander y actual maestrante en Semiótica de la misma institución. Docente de Lenguaje en la Universidad Industrial de Santander, Colombia, y miembro del grupo de investigación "Cultura y Narración en Colombia" (CUYNACO) en la línea de Prácticas educativas, cuya área de interés es el análisis de los procesos educativos desde las ciencias del lenguaje y la educación. 


\title{
Resumen
}

Este artículo, sobre el discurso pedagógico de los maestros en colegios privados y católicos, hace parte de la investigación Representaciones de la educación formal y la escuela en estudiantes de colegios privado-católicos del área metropolitana. Para su desarrollo se toman enunciados de educandos pertenecientes a algunas instituciones católicas, donde narran sus experiencias y percepciones académicas en el aula de clase. Luego se realiza un análisis discursivo apoyado en el diálogo con los postulados teóricos de Basil Berstein, Mario Díaz, Julius Greimás, Jackes Fontanille y Paulo Fabbri. Como resultado se defiende la idea, desde los educandos, de la presencia en los docentes de dos tipos de discurso pedagógico, uno donde lo confesional está permeando totalmente lo académico y otro donde lo académico se desliga de lo confesional. Se espera que este trabajo contribuya a la discusión en torno de los discursos pedagógicos presentes en algunas aulas Colombianas.

Palabras clave: Discurso Pedagógico, dispositivo pedagógico, discurso instruccional, discurso regulativo.

\section{Pedagogic discourse in teachers from the utterances of students of catholic schools}

\begin{abstract}
This article, about the pedagogical discourse of teachers in Catholic and private schools, is approached from the research Representations of formal education and school students in Catholic private schools in the metropolitan area. For the development of this text is part of the statements of students at Catholic private schools, in this they tell us about academic experiences and perceptions in the classroom and this is oriented a dialogue with the theoretical postulates of Basil Bernstein, Mario Diaz, Julius Greimas, and others. As a result the idea advocated in the presence of teachers from two types of pedagogical discourse statements, one where the confessional is totally permeating academics and one with a clear separation thereof. This text is expected to contribute to the discussion on the educational reality of young people in some educational institutions in Colombia.
\end{abstract}

Key words: Pedagogic discourse, pedagogic device, instructional discourse, regulative discourse. 


\section{Discours pédagogique chez des enseignants, dès l'énoncé fait par des élèves des établissements scolaires catholiques}

\section{Résumé}

Cet article sur le discours pédagogique des enseignants dans des établissements scolaires privés et catholiques, fait partie de la recherche Représentations de l'éducation formelle et l'école chez des élèves des établissements scolaires privés catholiques dans la zone urbaine. Pour son développement on prend des énoncés des apprenants appartenant à quelques institutions catholiques, où ils racontent leurs expériences et perceptions académiques dans la salle de classe. Ensuite, on fait une analyse discursive du dialogue avec les postulats théoriques de Basil Berstein, Mario Díaz, Julius Greimas, Jackes Fontanille et Paulo Fabbri. Comme résultat, partant des apprenants, on défend l'idée de la présence chez les enseignants, de deux types de discours pédagogique: l'un où le confessionnel rend perméable ce qui est académique ; l'autre où l'académique se détache de ce qui est confessionnel. On espère que ce travail contribuera à la discussion autour des discours pédagogiques présents dans quelques salles de classe colombiennes.

Mots clés: Discours Pédagogique, dispositif pédagogique, discours d'instruction, discours de régulation.

\section{Discurso pedagógico em professores desde o enunciado por estudantes de colégios católicos}

\section{Resumo}

Este artigo, sobre o discurso pedagógico dos professores em colégios privados e católicos, faz parte da pesquisa Representaciones de la educación formal y la escuela en estudiantes de colegios privado-católicos del área metropolitana. Para seu desenvolvimento se tomam enunciados de educandos pertencentes a algumas instituições católicas, onde narram suas experiências e percepções acadêmicas na sala de aula. Logo se realiza uma análise discursiva apoiada no diálogo com os postulados teóricos de Basil Berstein, Mario Díaz, Julius Greimás, Jackes Fontanille e Paulo Fabbri. Como resultado se defende a ideia, desde os educandos, da presença nos docentes de dois tipos de discurso pedagógico, um onde o confessional está permeando totalmente 0 acadêmico e outro onde 0 acadêmico se desliga do confessional. Espera-se que este trabalho contribua à discussão no que é referido aos discursos pedagógicos presentes em algumas salas Colombianas.

Palavras chave: Discurso Pedagógico, dispositivo pedagógico, discurso instrucional, discurso regulamentar. 
"No existen prácticas sin sujetos y no existen sujetos sin discursos y textos."

Basil Bernstein y Mario Díaz

\section{Introducción}

La educación, como fenómeno propio de la cultura, contempla diversos proyectos para formar a los jóvenes desde distintas líneas de orientación en el país, para responder a intencionalidades precisas, por lo que es posible encontrar la educación pública, la militar y la confesional, entre otras. Todas capaces de crear universos simbólicos en el educando que influyan en su ser, sentir y actuar, es decir, manipulan la construcción de significados, la adquisición de competencias e instauran en los jóvenes orientaciones de valor. En pocas palabras, tienen la capacidad de fortalecer, cambiar o instaurar, a través de los sujetos, formas de vida específicas. Para el caso de este análisis, es necesario reconocer que una educación confesional presenta sus propias intencionalidades educativas guiadas por una axiología particular presente y evidenciable, en el diario vivir de los actores principales en las escuelas, los educandos, pues ellos ingresan a las instituciones y experimentan una gran diversidad de prácticas coherentes con la forma de vida confesional.

En Colombia este tipo de educación siempre ha estado presente en la historia de todo el territorio y en pleno siglo XXI continúa vigente en 29 departamentos como lo muestra en su página web la Confederación Nacional Católica de Educación (CONACED). Esto indica que una gran población de jóvenes, en edad educativa básica, está inmersa en un discurso pedagógico que responde a unas axiologías determinadas, por lo que es pertinente identificar la forma como se constituyen y reproducen en la práctica estos discursos. La hipótesis es que existen dos discursos básicos, uno es el académico propio de asignaturas como biología, filosofía o español y el otro de orden confesional presente en áreas como religión o ética. Sin embargo, el punto en que se relacionan o separan es solo evidenciable a través del análisis. 
Por tanto, en el presente artículo se analiza el discurso pedagógico de los maestros desde la visión construida por los educandos en los colegios católicos del área metropolitana de Bucaramanga (Colombia) al que pertenecen. La importancia de estos enunciados parte del reconocimiento de que los involucrados en el proceso educativo (estudiantes), presentan un universo simbólico resultante del mundo de la vida que experimentan en estos centros educativos.

En este sentido, desde la perspectiva del análisis discursivo, los aportes del estudio desarrollado son importantes de cara a establecer una comprensión sobre los discursos pedagógicos que llegan a los jóvenes durante su formación académica y confesional en la escuela católica. Además, permitirá establecer una relación directa entre las acciones que ocurren en el interior de las aulas y lo reflejado fuera de ellas por los diversos actores involucrados en este tipo de proceso educativo, logrando así ampliar la frontera del conocimiento sobre el tema de la enseñanza-aprendizaje e iniciar con una posible innovación del análisis del mismo en correlación con los postulados educativos católicos.

\section{Del proceso metodológico}

Desde los postulados pedagógicos paidocentristas, el educando es el centro del proceso educativo, por tanto, es sobre quien recaen los esfuerzos de mediación. Desde la mirada semiótica, y este estudio, es relevante centrar la atención en el sujeto, pues su existencia ocupa en los enunciados posiciones que corresponden tanto a sujetos de estado como a sujetos de hacer durante el proceso de formación, y es precisamente en estos estados y transformaciones percibidas por el mismo que es posible reconocer lo que es para el joven estudiante la educación y la escuela. Por tanto, hablar de educación formal y escuela, desde la perspectiva del educando es partir del conocimiento experiencial, es indagar en los imaginarios impregnados de vivencias, en unas formas de vida particulares donde se enuncia cómo los educandos se perciben en el proceso educativo y en la vida cotidiana escolar.

Por lo que para el desarrollo de este análisis se partió del discurso enunciado por 10 educandos de los grados $9^{\circ}, 10^{\circ}$ y $11^{\circ}$ de colegios privado-católicos, sobre temas como las formas de enseñanza de sus docentes, la relación y diferencia entre los aprendizajes considerados académicos y aquellos con un tinte confesional y la forma como en las aulas los docentes manejan un discurso que tiende a la secularidad o a lo confesional católico. Es necesario aclarar que todos los informantes han estudiado un largo tiempo de su vida en estas instituciones católicas, algunos desde el preescolar y otros desde la primaria. De esta manera, tras una serie de entrevistas semiestructuradas dirigidas a los educandos, se logra configurar el nivel de los textos-enunciados que no son más que el producto de 
prácticas significantes poseedoras de un acervo cultural donde la enunciación representa lo que se experimenta.

A partir del registro obtenido y de la variedad de definiciones aportadas en los enunciados, se procedió al análisis, desde diversas teorías semióticas, linguísticas y pedagógicas, con miras a hallar rasgos dominantes y concurrentes en los enunciados, que permitieran generalizar la información obtenida en categorías capaces de construir un metalenguaje para describir los discursos pedagógicos y, por ende, la cultura educativa presente en las representaciones que los educandos de $9^{\circ}, 10^{\circ}$ y $11^{\circ}$ han construido sobre los discursos presentes en profesores de las escuelas católicas a las que pertenecen.

\section{Acerca del Discurso pedagógico de la educación}

El discurso y su estudio presentan diversas dificultades generadas por la manera como se concibe o aborda el sentido de este concepto. Por tanto, es necesario dar cuenta de la perspectiva específica bajo la cual se da la comprensión del término en esta investigación. Así las cosas, es necesario retomar la idea de que hablar del discurso implica evitar la reducción del mismo a una simple realización del lenguaje y, en cambio, se debe reconocer como un mecanismo esencial que esquematiza globalmente nuestras representaciones y nuestras experiencias, es decir, que los procesos discursivos pueden considerarse como el producto de una red compleja de relaciones sociales (Bernstein \& Díaz, 1984).

Se hace indispensable ampliar la visión, no solo reconociéndolo como un conjunto de significados en relación con representaciones o referentes, sino como parte de una práctica social donde sistemáticamente se da una intervención del discurso para construir y transformar, pero, a su vez, es constituido y transformado. Foucault (1972) plantea que en esa relación discurso y prácticas discursivas existe una cantidad de procedimientos relacionados con el poder, que controlan el discurso desde el campo social a través de la exclusión, la prohibición, la división y la distinción, en otras palabras, estos sistemas de control operan desde fuera del discurso.

Así, en una práctica social el proceso de enunciación se considera allí como una actividad discursiva sustentada y constituida por un sistema de mecanismos institucionalizados, estatutos y posiciones diferentes asignadas al sujeto en el discurso y que este, de forma consciente 0 inconsciente, asume. Fontanille (2008) plantea la importancia de reconocer en el discurso la enunciación en acto donde el enunciado (producto) no puede ser disociado en su análisis de la enunciación (la producción), debido a que es 
en esta última donde se identifica la instancia de discurso ${ }^{1}$, que de forma amplia abarca la perspectiva, influencias sociales, poderes y procedimientos que permean la relación discurso y práctica.

En consecuencia, es posible afirmar que el hablante no produce el sentido de manera libre, sino que está sujeto a un orden discursivo en el cual está ubicado y en el cual ubica sus enunciados; siguiendo a Benveniste (1996, p. 192) está en "un campo posicional". Este orden discursivo se encuentra inmerso en unas demandas del orden no discursivo (orden dominante social) en el cual está ubicado y al cual reproduce debido a la presencia de unas fuerzas de poder que Bernstein y Díaz $(1984$, p. 3) reconocen cuando plantean que "el poder está presente en cada discurso, y, a su vez, cada discurso es un mecanismo de poder". Se piensa el discurso como una categoría que implica relaciones de poder y control alrededor de la actividad y los actos semióticos que involucran la producción discursiva en las diversas prácticas sociales.

En este sentido, más que la producción lingüística, es la producción de un orden, la constitución de una conciencia desde los procesos sémicos en el interior de los universos simbólicos de los sujetos, lo que resulta ser la tarea específica de ciertos tipos de discursos. Precisamente, por su característica particular de poder ser constituido por otros discursos y, a la vez, ser el medio y la fuente de producción de nuevos discursos. Frente a esto último, Foucault (1972) considera que cualquier sistema, en especial el educativo, es una forma política de mantener o modificar la apropiación de los discursos con los saberes y poderes que implican, para lo que utiliza una forma especializada de comunicación como lo es el discurso pedagógico de la educación.

Este tipo de discurso, que responde a un proyecto y unos fines educativos de los distintos estados, es producto de un mecanismo de poder y de control simbólico ${ }^{2}$ para las posiciones de los sujetos en órdenes específicos, por tanto, puede considerarse como un dispositivo de reproducción de formas de conciencia específica que responde a la producción de unas reglas determinadas y, a su vez, regula las relaciones sociales entre categorías específicas como transmisor y adquirientes. En consecuencia, controla los significados que se realizan en la práctica pedagógica al regular las posiciones de los

1 Para Fontanille, la instancia de discurso o discurso en acto comporta un pequeño número de propiedades como una posición, un campo, actantes, posiciones deícticas y operaciones, es decir, un conjunto de operaciones, operadores y de parámetros que controlan y organizan la enunciación y la perspectiva o el campo desde el que se enuncia.

2 Es indispensable reconocer este poder y control simbólico no necesariamente de manera negativa, sino como parte de una estrategia de formación del individuo según las necesidades y prioridades de los estados y, por supuesto, la ideología particular del momento histórico, donde se trata de distribuir el poder, y las categorías culturales dominantes se legitiman, mantienen, desarrollan o cambian. 
sujetos y de las relaciones sociales en el contexto institucional organizado del sistema educativo que Bernstein (1984, p. 17) denomina "contexto de reproducción"3.

Es evidente la diferencia entre la estructura macro-institucional de la generación del discurso pedagógico (contexto de producción) y el orden que este produce (contexto de reproducción) en el conocimiento educativo. Entonces, el discurso pedagógico puede entenderse como las reglas que codifican, por un lado, producción, y por otro, la distribución, reproducción, interrelación y cambio de lo que cuenta como textos pedagógicos legítimos, debido a que no sería un repertorio de contenidos (conceptos o teorías), sino una gramática, similar a una región, que selecciona no solamente del campo total de producción del discurso de diversas disciplinas, sino también del campo de las prácticas. En palabras de Bernstein (1990, p. 143), "El Discurso Pedagógico es un discurso sin discurso específico; no tiene un discurso propio", pues se compone de otros discursos especializados. En este sentido, es importante considerar cómo está constituido el Discurso Pedagógico y cómo se convierte en una categoría estructurante y subyacente del contexto de reproducción propio del nivel de las prácticas pedagógicas en las instituciones educativas.

Así las cosas, es posible analizar el tipo de discurso pedagógico presente en la educación formal de las escuelas con proyectos específicos de formación como el de las instituciones privado-católicas. Para lo cual, como ya se anunció, se parte de las entrevistas realizadas a un grupo de jóvenes de $\operatorname{los}$ grados $9^{\circ}, 10^{\circ}$ y $11^{\circ}$, sobre su personal experiencia en las prácticas pedagógicas en el interior de las aulas; cabe advertir que se involucra, como actores esenciales del proceso, tanto al docente como al educando en el espacio de las aulas.

\section{El discurso pedagógico en el contexto de reproducción de algunas instituciones privado-católicas}

El discurso pedagógico presenta una normatividad interna que se denomina "dispositivo pedagógico" reproducción específico en el que es posible encontrar unas interrelaciones entre agentes, unos discursos (instruccionales y regulativos), prácticas y sitios articulados para lograr la reproducción de esas formas especializadas del discurso. Para el análisis, es necesario reconocer que los tres colegios participantes manejan un horario regular en el que se

3 Bernstein reconoce la presencia de dos campos relacionados con el discurso pedagógico. El primero, denominado contexto de producción y el otro, Contexto de reproducción.

4 El dispositivo pedagógico, término propuesto por Bernstein, enmarca una gramática interna del ordenamiento del discurso pedagógico. 
encuentran las distintas asignaturas como Física, Química, Historia, Español, etc. Es decir, manejan el currículo básico en cumplimiento de lo exigido por la Ley General de Educación, $n^{\circ} .115$ de 1994.

En esta medida, los colegios de educación formal privado-católicos presentan unos discursos pedagógicos de los maestros, según la experiencia personal de los educandos, cargados de elementos instruccionales y regulativos específicos evidentes en las prácticas educativas. De esta manera, se muestran los siguientes enunciados que surgen de la pregunta ¿Está la creencia católica afectando o involucrada en lo que usted aprende en las distintas asignaturas?

Colegio A: (Interviene un estudiante) [...] acá nos muestran de todo lo de la realidad [...] aqui vemos cualquier tema sin ningún problema. Con el padre de religión (el profesor es un sacerdote) vemos por ejemplo lo de los exorcismos y es lo más interesante [...] y lo de los exorcismos va de la mano de lo religioso porque tiene una serie de pasos que siempre tiene que estar confesado y ser buena persona porque los demonios le van a reconocer los pecados y todo eso. Por ejemplo un tema de sexualidad que en otro colegio de pronto se toca como aquí somos solo hombres solo se habla de la prevención.

Colegio B: (Interviene un estudiante) [...] la verdad es que ayuda (lo católico) en la parte de valores en lo de la persona y cómo ve las cosas [...] pero se ve todo como es en los libros y como lo encuentra en internet. Acá los profesores nos lo explican todo, o sea, no hay censura generadas por la religión o no nos impiden bablar de ciertos temas en clase por la religión o no nos impiden debatir por la religión sobre temas de Dios o teorías del libro, nada se impide todo se permite a pesar de que se maneja la religión católica y, a pesar de que tenemos clases especificas para ver y estudiar nuestra religión, también estudiamos otras religiones y vemos cómo nos aporta la religión católica a nuestra moral y nuestros valores.

(Interviene otro estudiante) [...] hay profesores que son católicos y que al comienzo de la clase intentan dar como una pequeña oración o algo así y ya en el transcurso del día es lo normal, o sea, los temas que debemos ver [...]

Los enunciados anteriores permiten observar que el campo católico regula el discurso pedagógico de diversas formas en las instituciones y durante la práctica pedagógica. Así, los dos primeros colegios (A y B) dejan ver que en el horario de clase tienen momentos específicos para la vivencia de lo confesional; por un lado, la clase de religión establecida para aprender y afianzar conocimientos sobre el marco Católico y por otro, el inicio de las clases generales (Física, Español, Historia, etc.) con oraciones o reflexiones que 
promueven el pensamiento y la formación de individuos desde la perspectiva de la moral y la ética de la religión. En consecuencia, según lo enunciado por los informantes, la formación en conocimientos ${ }^{5}$ está desligada de la regulación confesional y precisan una clara diferencia entre el momento de adquirir conocimiento científico y el de adquirir conocimiento religioso.

Lo planteado hasta el momento por los educandos sobre los discursos pedagógicos de sus docentes, disgrega los imaginarios comunes que tienden a creer, sin duda alguna, que todo profesor perteneciente a una institución católica impregna su hacer de los valores culturales de la comunidad a la que pertenece y, por tanto, no enseña según la rigurosidad de cada disciplina. Lo anterior es posible, pues los docentes de estas instituciones han sido formados en distintos entornos académicos como lo mencionan los estudiantes, [...] acá tenemos profesores que son licenciados, creo que el de Matemáticas es uno, otros son ingenieros como el de Química y así [...]. Es entonces evidente una competencia cognitiva en los docentes que los faculta para la enseñanza rigurosa de cada asignatura, aunque no necesariamente desligada por completo de la ideología confesional.

Por otro lado, es posible encontrar una perspectiva un tanto diferente en el colegio C, como se muestra a continuación:

Colegio C: (Interviene un estudiante) [... I Influye en la manera de enseñar porque bay colegios donde exigen más y pues el trato con los estudiantes es más fuerte, en cambio acá como es un colegio católico, privado y todo eso, obviamente los profesores no nos pueden tratar fuerte porque ya iría con problemas de lo social a rectoría, entonces eso es lo que más influye, la manera de tratarnos...

Partiendo de lo anterior, se puede afirmar que en los dos últimos colegios (B y C) el comportamiento de los sujetos (maestros y estudiantes), en el marco de la actuación, se encuentra condicionado por el discurso religioso y las formas de comportamiento esperadas por el mismo en estos actores; por ejemplo, se les exige evitar los fraudes, mantener buenas relaciones entre todos, vivir los valores, mirar las cosas de la vida desde la perspectiva de un creyente y reflexionar sobre los actos. En otras palabras, a nivel procedimental y valorativo, hasta el momento, el discurso regulativo es de tipo confesional.

Se está, entonces, ante sujetos constituidos entre las relaciones de poder de una autoridad confesional y unos principios de control de los procesos sociales y los

5 El Ministerio de Educación Nacional de Colombia (MEN) propone una formación en competencias para el país; plantea que el término competencia debe ser entendido como un elemento que integra conocimientos, habilidades y valores; es decir, comprende aspectos de tipo cognitivo, procedimental y actitudinal. 
comportamientos desde la misma figura. Es evidente que el poder del régimen de creencia católica está presente en cada discurso de los estudiantes, pero cada discurso ha sido construido de tal manera que les permite a los jóvenes dar cuenta de cuándo están ante una situación de orden católico y cuándo ante una que no se le asemeja por ser de un orden más crítico y racional, académico.

\section{Discurso regulativo vs. Discurso instruccional}

El discurso según Fabbri (1979), en especial el didáctico, es una estructura formal que subsume objetos cognitivos diversos que son manipulados, es decir, seleccionados y organizados, luego, modalizados y axiologizados, estableciendo nuevas relaciones jerárquicas y organizando su propio universo doxológico. Por tanto, todo discurso pedagógico se compone de una base instruccional y una regulativa que le configura, legitima y da sentido en las realizaciones de la práctica organizativa.

Pero para saber cómo se da en las instituciones católicas esta relación del discurso instruccional y el regulativo, se parte de la siguiente pregunta ¿Cómo se involucra la enseñanza católica en asignaturas que usted aprende como Biología, Historia, Español, Química, entre otras que no son netamente religiosas? Y se obtuvieron los siguientes enunciados de los educandos:

Colegio A: (Interviene un estudiante) [...] No se ve lo religioso. Por ejemplo en la evolución una cosa es lo de Dios y la evolución es otra cosa, entonces desde Dios, él creó al hombre y lo de la evolución si es ya que nosotros, el ser humano actual, tenemos antepasados similares al mono, entonces que a medida del tiempo salimos nosotros. No bay mezcla...es más académico que religioso...

Colegio B: (Interviene un estudiante) [...] Las asignaturas se enseñan normal, no tiene nada que ver que el colegio es católico pero las clases se dan normal, digamos en Biología en la célula no implica necesariamente a Dios, o sea todo es independiente, a nosotros nos dan unas teorías y nosotros debemos escoger lo que queremos, nos basamos más en lo científico independientemente de Dios.

La producción de un orden, la constitución de una conciencia específica, que construye, moldea o modifica el universo simbólico de los sujetos, es la tarea de ciertos tipos de discursos. El pedagógico es un ejemplo claro, pues puede considerarse como un dispositivo de reproducción de formas de conciencia específica. Para el caso de los colegios anteriores, los enunciados plantean una formación, por parte de los docentes, que desarrolla la visión crítica del educando, pues le permite conocer y afirmar el conocimiento científico sin ningún detrimento por causa del conocimiento religioso, ni de 
las reglas específicas que regulan procedimental y valorativamente las relaciones sociales en el interior de las aulas y la misma institución. De igual manera, es evidente que la ubicación del sujeto es una ubicación en principios dominantes (privilegiantes), es decir, se forma para lograr sujetos autónomos y libres, capaces de reconocer y apropiarse de lo mejor de ambos tipos de conocimiento.

Por su parte, el colegio C ubica a los sujetos en el principio de dominados (no privilegiantes), pues no permite la diferenciación entre lo científico y lo religioso. En consecuencia, los docentes plantean una clara aceptación e imposición de las creencias confesionales, por encima de las científicas o filosóficas que vayan en contra de los postulados católicos. Se muestra la clara disforia por estos pensamientos; veamos:

Colegio C: (Interviene un estudiante) Yo pienso que eso depende del profesor porque los profesores son los que van a explicarle a uno y si ellos son digamos ateos aunque estén en un colegio religioso pues ellos no van a manifestar de la misma forma como un profesor que sea católico, entonces cuando son religiosos católicos, pues le enseñan a uno también por ese lado de los buenos valores, de estar por el camino del bien y esas cosas, entonces más que por el área es por lo que el profesor su religión y eso [...] en filosofía cuando bablamos de los filósofos, hay muchos que plantean que Dios no existía y por más de que esas teorías las explique mi profesor es católico, entonces obviamente va a ir siempre en contra, bueno no en contra, solo que no lo manifiesta bien y siempre pone lo religioso, entonces todo está encadenado bacia lo religioso a que Dios si existe por más de que sea

(Interviene otro estudiante) por ejemplo en la constitución del mundo de la parte de Biología, que la profesora también es bastante devota, ella nos explica la teoría científica de la creación del mundo y todo eso, pero también lo de la parte del alma y todo eso, que pues si viene influenciada.

Entonces, el discurso regulativo de este colegio permea totalmente el discurso instruccional; por tanto, se puede afirmar que es este primero el que constituye el dispositivo dominante para la regulación de la reproducción cultural y educativa católica en el universo simbólico de los educandos y los coarta del acceso a una formación crítica frente a lo que es el conocimiento y sus formas de construirse en la vida real.

Se obtiene, entonces, que entre un conocimiento religioso y uno científico-filosófico está la diferencia entre una educación que tiende a promover el pensamiento críticoracional y el pensamiento mítico ${ }^{6}$. Entonces, según los enunciados de los estudiantes, se

6 Eduardo Laso (1995) plantea que en el pensamiento mítico las relaciones de fuerza, autoridad y sumisión ante los dioses son los 
puede afirmar que para trascender el conocimiento de lo religioso, es necesario fomentar un conocimiento analítico y crítico en el educando que le permita llegar a un conocimiento científico-filosófico más allá de los dogmas; y dar un conocimiento académico sin lo analítico es caer de nuevo en un pensamiento mítico, como se observa en la siguiente figura (o ilustración) denominada cuadrado semiótico de la formación del pensamiento en las instituciones privado católicas a través del discurso pedagógico de los docentes:
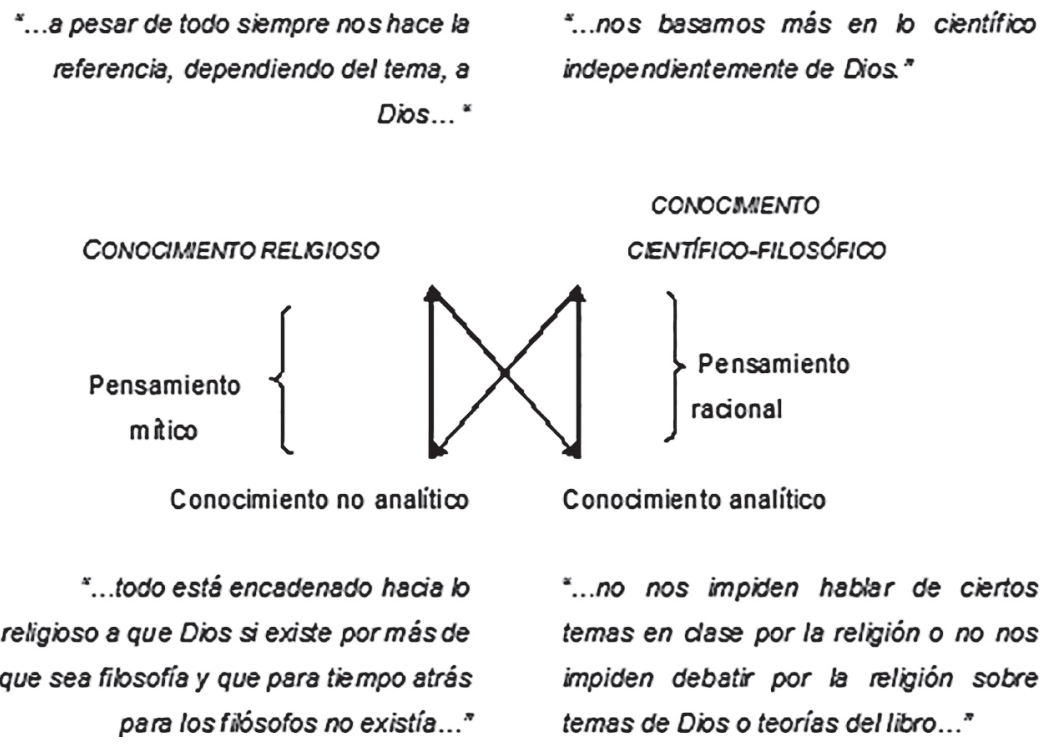

\section{Ilustración 1. La formación del pensamiento en las instituciones privado-católicas}

Es evidente que el discurso pedagógico de los docentes en las instituciones católicas es diferente y responde en gran medida al campo regulativo del discurso que Bernstein (1990) asocia con la voz silenciosa en el interior de la comunicación, pero que tiene todo el manejo del poder durante el proceso de realización y reproducción educativa.

\section{Conclusión}

El discurso pedagógico, en lo referente a lo cognitivo de los colegios privadocatólicos, presenta dos formas evidentes según lo enunciado por los educandos. Entonces, se tiene un discurso pedagógico donde lo académico, es decir, lo instruccional, encargado de transmitir habilidades especializadas en el conocimiento científico-filosófico de un 
área específica, resulta dominado por lo regulativo del pensar católico en las condiciones sociales para su reproducción y termina configurando una institución tradicionalista, es decir, basada en la tradición y el pensamiento mítico. Entonces, se tiene el siguiente esquema:

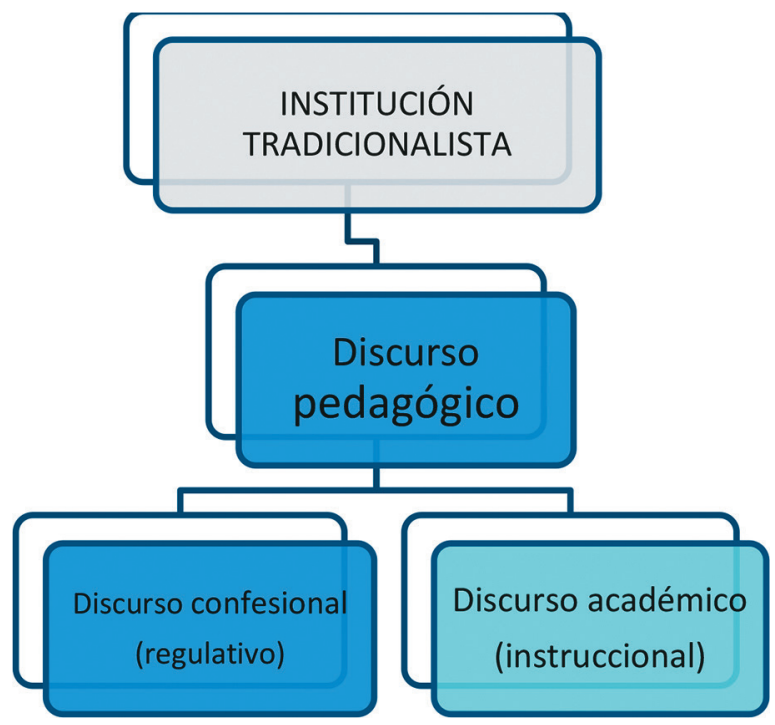

\section{Ilustración 2. El discurso pedagógico en la institución Tradicionalista}

Este tipo de discurso pedagógico, parte de un conocimiento fundamentalmente religioso en el que todo se explica desde la figura de Dios como sujeto principal en el acceso al discernimiento de las percepciones, es decir, se encuentra fundamentado en la creencia, la fe y el dogmatismo no metódico que lleva al desarrollo de un conocimiento religioso que promueve el pensamiento mítico, en contraposición a la era de la ciencia y la razón que viven los estudiantes en pleno siglo XXI. Esto explica la razón por la que los educandos del Colegio $\mathrm{C}$ enuncian de manera disfórica la forma como sus docentes les enseñan prestando más atención a sus ideologías confesionales, que al rigor disciplinar.

En contraposición, aparece un discurso pedagógico donde se dan dos esferas definidas de acción. Por un lado, lo netamente académico con su regulación propia, desde el ámbito de la cultura del conocimiento, con sus principios de criticidad, veracidad y relatividad y, por otro, el conocimiento religioso con su regulación confesional y de fe. Como se muestra a continuación: 


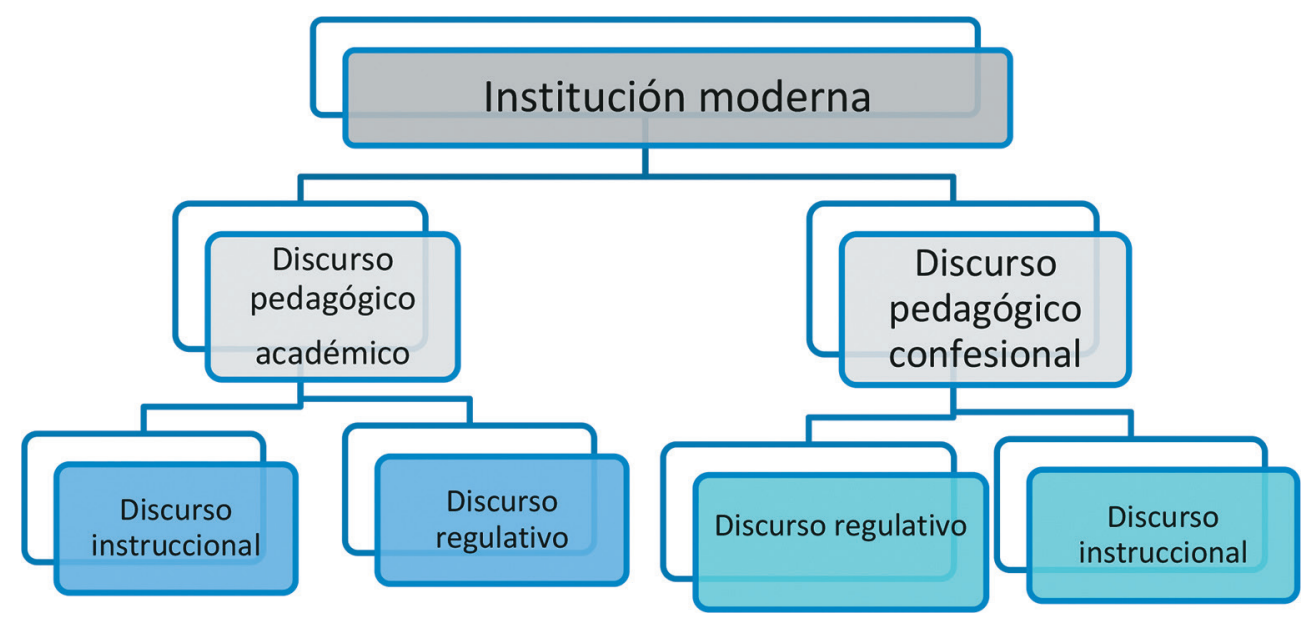

Ilustración 3. El discurso pedagógico en la institución moderna

La manera de desarrollar los proceso formativos en este tipo de discurso es orientado por un proceso más analítico, riguroso y de investigación. El educando será capaz de acceder al conocimiento científico, cuyo valor de veracidad, aunque relativo, es más objetivo y tiende al desarrollo del pensamiento racional donde el centro es el sujeto mismo (ser humano como ser racional); pero sin perder la noción ideológica de la fe que profesan. Este discurso pedagógico permite al estudiante contrastar el mundo cultural y social al que pertenece, mientras toma lo mejor de ambos tipos de pensamiento, para acercarse a las formas como el ser humano ha adquirido conocimiento de su mundo a través de su existencia histórica.

Finalmente, desde la formación en el campo procedimental y valorativo es claro que el discurso pedagógico recibido por el educando no muestra diferencias entre lo instruccional y lo regulativo, por el contrario, los principios y valores confesionales mantienen el poder desde el campo regulativo. Es claro que el educando se encuentra en medio de este juego del desarrollo de pensamientos, mítico y racional, en las formas de vida de la educación católica, pero depende de cada institución decidir a cuál se le da prioridad y cómo lo hace. 


\section{Referencias bibliográficas}

Benveniste, E. (1996). Problemes de linguistique générale I. París. Traducido al español como Problemas de Lingüística general I. México: Siglo XXI.

Berstein, B., \& Díaz, M. (1984). Hacia una teoría del discurso pedagógico. Traducido de Collected Original Resources in Education (CORE), 8 (3).

Berstein, B. (1990). La construcción social del Discurso pedagógico, Bogotá: El Griot.

Fabbri, P. (1979). Champ de manoeuvres didactiques. Publicado originalmente en Le Bulletin, $\mathrm{n}^{\circ} 7$, París, editado por Groupe de Recherches sémiolinguistiques (EHESS) y el Instituto de la Langue Francaise (CNRS).

Fontanille, J. (2008). Textos, objetos, situaciones y formas de vida: los niveles de pertinencia de la semiótica de las culturas. Traducción de Horacio Rosales, Bucaramanga: Colombia, junio 2010. De la obra original de Fontanille (2008). Practiques semiótique. París: PUF.

Foucault, M. (1972). The Archaeology of Knowledge. London: Tavistock Publications.

Ministerio de Educación Nacional de Colombia. Fundamentos conceptuales. Recuperado de http://www.mineducacion.gov.co/1621/w3-printer-299611.html

Laso, E. (1995). Pensamiento mítico y pensamiento racional. En AAV: La producción de los conceptos científicos. Esther Díaz, compiladora. Buenos Aires: Biblos. 